\title{
Longevity in the red flour beetle Tribolium castaneum is enhanced by broccoli and depends on $n r f-2$, jnk-1 and foxo-1 homologous genes
}

\author{
Stefanie Grünwald $\cdot$ Julia Stellzig $\cdot$ Iris V. Adam $\cdot$ Kristine Weber $\cdot$ Sarai Binger $\cdot$ Michael Boll $\cdot$ \\ Eileen Knorr · Richard M. Twyman · Andreas Vilcinskas · Uwe Wenzel
}

Received: 18 October 2012/Accepted: 31 December 2012/Published online: 16 January 2013

(C) Springer-Verlag Berlin Heidelberg 2013

\begin{abstract}
Diet is generally believed to affect the aging process. The effects of complex foods on life span can be investigated using simple models that produce rapid results and allow the identification of food-gene interactions. Here, we show that $1 \%$ lyophilized broccoli, added to flour as a dietary source, significantly increases the life span of the red flour beetle (Tribolium castaneum) under physiological conditions $\left(32{ }^{\circ} \mathrm{C}\right)$ and under heat stress $\left(42{ }^{\circ} \mathrm{C}\right)$. The beneficial effects of broccoli could also be reproduced by supplementing flour with the isothiocyanate sulforaphane at concentrations found in the broccoli-supplemented diet. We identified stress-resistant genes responsible for these effects on longevity by microinjecting pupae with double-stranded RNA to induce RNA interference (RNAi). The knockdown of transcripts encoding homologs of Nrf-2, Jnk-1 and Foxo-1 reduced the life span of beetles and abrogated the beneficial effects of broccoli, whereas the knockdown of Sirt-1 and Sirt-3 had no impact in either scenario. In conclusion, $T$. castaneum is a suitable model organism to investigate food-gene interactions that affect stress resistance and longevity, and RNAi can be used to identify functionally relevant genes. As a proof of principle, we have shown here that broccoli increases the longevity of beetles and mediates its effect through
\end{abstract}

\footnotetext{
S. Grünwald · J. Stellzig · I. V. Adam · K. Weber - S. Binger · M. Boll · U. Wenzel ( $\square)$

Molecular Nutrition Research, Interdisciplinary Research Center, Justus-Liebig-University of Giessen, Heinrich-Buff-Ring 26-32, 35392 Giessen, Germany

e-mail: uwe.wenzel@ernaehrung.uni-giessen.de

E. Knorr · R. M. Twyman · A. Vilcinskas

Applied Entomology, Interdisciplinary Research Center, Justus-Liebig-University of Giessen, Heinrich-Buff-Ring 26-32, 35392 Giessen, Germany
}

signaling pathways that include key stress-resistant factors such as Nrf-2, Jnk-1 and Foxo-1.

Keywords Longevity $\cdot$ Stress resistance $\cdot$ Tribolium castaneum - Food-gene interactions · Isothiocyanates

\section{Introduction}

A reduction in calorific food intake without malnutrition, described as caloric restriction or dietary restriction, has been widely accepted as an intervention to slow the process of aging (Fontana et al. 2010). Increased longevity in response to caloric restriction has been observed in diverse organisms ranging from yeast to mice (Masoro 2005) and appears to extend to primates including humans (Anderson et al. 2009; Anderson and Weindruch 2012; Fontana and Klein 2007). However, voluntary prolonged caloric restriction in humans is met with low compliance, so alternative strategies that support healthy aging are necessary. In this regard, it is important to identify dietary factors that promote health and longevity (Mattson 2008).

A number of phytochemicals appear to promote longevity by inducing the expression of genes encoding cytoprotective proteins, such as chaperones or antioxidant enzymes (Mattson 2008). Subtoxic doses of such phytochemicals, as achieved by the ingestion of plant-based foods by humans, generate mild stress that ultimately leads to an adaptive or hormetic response (Son et al. 2008). For example, isothiocyanates (which are present at high levels in broccoli) activate Nrf-2, a transcription factor that induces genes with antioxidant response elements in their promoters, such as those encoding antioxidant proteins or phase II detoxifying enzymes (Dinkova-Kostova et al. 2002; McWalter et al. 2004). Similarly, resveratrol (which 
is present at high levels in the skin of red grapes and in red wines) activates sirtuins, which are key factors mediating the effects of caloric restriction on hormesis and longevity (Chen and Guarente 2007; Frescas et al. 2005). Accordingly, the effect of selected phytochemicals and caloric restriction appears to be integrated by inducing mild stress, and even in humans, it has been shown that blocking exercise-induced stress efficiently abolishes hormetic adaptations (Ristow et al. 2009).

The effects of dietary factors on aging and longevity can be investigated using simple models, which can also be used to determine the underlying mechanisms. However, classical models such as the nematode Caenorhabditis elegans are hampered by their eating habits, which prevent the standardized intake of complex foods. We therefore used the red flour beetle (Tribolium castaneum) to determine the effects of lyophilized broccoli, added to the normal flour diet at concentrations of $0.1-10 \%$, on survival and longevity under physiological conditions $\left(32{ }^{\circ} \mathrm{C}\right)$ and under heat stress $\left(42^{\circ} \mathrm{C}\right)$. So far, Tribolium has been suggested more than 25 years ago to be a proper model to study age-related changes and parental age effects (Soliman 1987) or age-associated increase in oxygen sensitivity (Lee and Ducoff 1983). Only one recent study has picked up this knowledge to demonstrate that genotype-by-environment interactions may be important in facilitating adaptation to new environments and maintaining genetic variation in male traits (Lewis et al. 2012).

We here also used RNA interference (RNAi) to investigate the relevance of different stress-response genes in the context of broccoli-mediated longevity under heat stress. Since the T. castaneum genome has been fully sequenced (Richards et al. 2008), we were able to construct PCRamplified cDNAs with T7 extensions to allow the synthesis of dsRNA for microinjection by in vitro transcription.

\section{Methods}

Tribolium castaneum strains and maintenance

Wild-type Tribolium castaneum strain San Bernardino and GFP recombinant strain Pig-19 (Lorenzen et al. 2003) were reared in darkness on a whole-grain flour diet supplemented with $5 \%$ yeast powder (basal medium) on glass Petri dishes $\left(20 \mathrm{~cm}\right.$ diameter) at $32{ }^{\circ} \mathrm{C}$ and $70 \%$ relative humidity, as described elsewhere (Altincicek et al. 2008).

\section{Broccoli supplements}

Fresh broccoli florets (Brassica oleracea) were cut into small pieces and lyophilized overnight and then ground to fine powder using a mortar and pestle. We mixed
1-100 mg of powder with enough basal medium to make the total weight up to $1 \mathrm{~g}$, resulting in final concentrations of $0.1-10 \%(\mathrm{w} / \mathrm{w})$. The medium was mixed thoroughly to ensure an even distribution of the vegetable powder.

The same procedure was used to spike the feed with isolated isothiocyanates (Sigma, Deisenhofen, Germany) except that the fine chemicals were first dissolved in $70 \%$ ethanol to make $0.2-20 \mu \mathrm{g} / \mathrm{ml}$ stocks, $5 \mathrm{ml}$ of which was pipetted onto $6-\mathrm{cm}$ Petri dishes loaded with $1 \mathrm{~g}$ basal medium to achieve final concentrations of $0.0001-0.01 \%$ $(\mathrm{w} / \mathrm{w})$. The liquid was allowed to evaporate overnight under a laminar flow hood and then ground to fine powder using a mortar and pestle. Control plates were treated with $70 \%$ ethanol only.

\section{Measurements of food intake}

Beetles were starved for $6 \mathrm{~h}$ before 30 beetles per group were randomly assigned to feed on one of the broccoli concentrations during 2,4 or $6 \mathrm{~h}$ either at $32{ }^{\circ} \mathrm{C}$ or at $42{ }^{\circ} \mathrm{C}$, after which individuals were frozen at $-18{ }^{\circ} \mathrm{C}$. Food intake was determined by measuring the wet weight of 5 individuals on a microbalance with 6 replicates per concentration per species.

\section{Life span determination}

Synchronized adult beetles were obtained by distributing 30 pupae per plate (two plates per condition) on Petri dishes (92 $\mathrm{mm}$ diameter) containing the basal medium (control) or basal media supplemented either with broccoli or with isothiocyanates. Pupae were hatched completely after 4 days, and beetles remained on the plates thereafter at $32{ }^{\circ} \mathrm{C}$ and $70 \%$ humidity. Beetles were transferred to freshly prepared media every 14 days and scored for dead beetles directly before the media changes.

For the thermotolerance assay, beetles were hatched and treated as described above. Media changes were done here every 7 days. After 14 days of feeding at $32{ }^{\circ} \mathrm{C}$, beetles were transferred to an incubator at $42{ }^{\circ} \mathrm{C}$ at $70 \%$ humidity, and henceforward, surviving animals were scored every $12 \mathrm{~h}$.

\section{Bacterial strains and GFP cloning}

Feeding with dsRNA-expressing bacteria is a standard protocol to achieve RNAi in C. elegans. Accordingly, we evaluated the efficiency of this method in $T$. castaneum by constructing an $E$. coli clone expressing GFP dsRNA to achieve GFP knockdown in the GFP-expressing T. castaneum strain Pig-19. The GFP-coding sequence from pPD95 75 (Addgene, Cambridge, UK) was inserted into the pGEM-T-easy vector (Promega, Mannheim, Germany) 
using the BamHI and ApaI sites and introduced into E. coli strain TOP 10F' (Invitrogen, Karlsruhe, Germany). A 225-bp insert was then transferred to vector L4440 (MRC GeneService Ltd., Cambridge, UK) and introduced into E. coli strain HT115 (DE3). The integrity of the construct was verified by custom sequencing with M13 and T7 sequencing primers (JLU, Microbiology Institute, Gießen, Germany). The expression of gene-specific dsRNAs was induced with $1 \mathrm{mM}$ isopropyl- $\beta$-D-thiogalactopyranoside (Sigma, Deisenhofen, Germany) for $1 \mathrm{~h}$ at $37{ }^{\circ} \mathrm{C}$ to trigger interference. The bacterial cells were then washed and $5 \times 10^{8}$ bacterial cells were mixed with $1 \mathrm{~g}$ of the basal flour medium after $1 \mathrm{~h}$ desiccation at $37^{\circ} \mathrm{C}$ or after lyophilization.

Generation of gene-specific RNAi constructs

Total RNA was prepared by grinding 200 beetles under liquid nitrogen in a mortar and using $200 \mathrm{mg}$ of powder for RNA extraction with the Roti-Quick kit (Roth, Karlsruhe, Germany) according to the manufacturer's instructions. We reverse transcribed $2 \mu \mathrm{g}$ of total RNA $\left(1 \mathrm{~h}\right.$ at $\left.37^{\circ} \mathrm{C}\right) \mathrm{using}$ the M-MLV Reverse Transcription kit (Promega, Mannheim, Germany) and gene-specific reverse primers for $n r f-2$ (Tc-nrf-2-B3356, accession number XM_963549; 5'-GCT ACA CAT TCA CAG GGA TAC-3'), jnk-1 (Tc-jnk-1B1172, accession number XM_969870; 5'-TAC CGC ATT GAA TCC GTC C-3'), foxo-1 (Tc-foxo-1-B1353, accession number XM_970107; 5'-ACT TCC CTC CAT ACT CAA CTC-3'); sirt-1 (Tc-sirt-1-B726, accession number XM_961975; 5'-CGC CAG TCG AAC GTA AAT CCC-3') and sirt-3 (Tc-sirt-3-B600, accession number XM_963962; 5'-CCT ACA CGC TAA ACA ATG TCC CG-3'). The number in each primer name corresponds to its annealing position within the corresponding cDNA.

A 2- $\mu$ l aliquot from each reverse transcription reaction was then used for PCR amplification with REDTaq DNA Polymerase (Sigma, Deisenhofen, Germany) and genespecific primers containing a flanking $\mathrm{T} 7$ promoter sequence (5'-TAA TAC GAC TCA CTA TAG GG-3') represented in the following sequences as ' $\mathrm{T} 7$ '. The primers for $n r f-2$ were Tc-nrf-2-F2587-T7 (5'-T7-TCG CCG TAC CAA TACAGT C-3') and Tc-nrf-2-B3091-T7 (5'-T7-GTC AGT CAC TCG CAT TCA TC-3'). Those for $j n k-1$ were Tc-jnk-1-F179-T7 (5'-T7-ATG TGA CGC ACG CTA AAA G-3') and Tc-jnk-1-B835-T7 (5'-T7-AGG GAA ACA GCA CAT CGG G-3'). Those for foxo-1 were Tc-foxo-1-F756-T7 (5'-T7-CAC CAC TCC TAG TCC TAG TTC-3') and Tc-foxo-1-B1095 (5'-T7-ATG CGG ATA CGA AGG CGA C-3'). Those for sirt- 1 were Tc-sirt1-F221-T7 (5'-T7-AGA CCT CAC CTC ACA CTG ACC-3') and Tc-sirt-1-B676-T7 (5'-T7-ACG AAA CAG AGA CTC CAG CGC C-3' ${ }^{\prime}$. And those for sirt-3 were Tc-sirt-3-F52-T7
(5' -T7-CCA GAA GCA CAG TCG GAG GAA G-3') and Tcsirt-3-B587-T7 (5'-T7-CAA TGT CCC GTG TAA AAA GTG CC-3' ${ }^{\prime}$.

Amplifications were carried out using a Thermocycler Primus 25 (peqlab, Erlangen, Germany) and each reaction comprised an initial denaturation at $94{ }^{\circ} \mathrm{C}$ for $2 \mathrm{~min}$ followed by 35 cycles of denaturation at $94{ }^{\circ} \mathrm{C}$ for $30 \mathrm{~s}$, annealing at $55^{\circ} \mathrm{C}$ (cycles $1-5$ ), $60{ }^{\circ} \mathrm{C}$ (cycles 6-10) or $65{ }^{\circ} \mathrm{C}$ (cycles $11-35$ ) for $30 \mathrm{~s}$ and extension at $72{ }^{\circ} \mathrm{C}$ for $90 \mathrm{~s}$ followed by a final elongation at $72{ }^{\circ} \mathrm{C}$ for $10 \mathrm{~min}$. PCR products were verified by agarose gel electrophoresis and inserted into vector pCR-2.1 using the TOPO-TAcloning kit (Invitrogen, Karlsruhe, Germany). Constructs were introduced into E. coli strain TOP10F' (included in the kit), and their integrity was verified by reamplification and sequencing.

dsRNA synthesis and injection

Gene-specific DNA templates in the pCR-2.1 vector were amplified by PCR using $10 \mathrm{ng}$ of the gene-specific RNAi plasmid and corresponding primer pairs as listed above. A fragment of the Galleria mellonella insect metalloproteinase inhibitor (IMPI) gene was amplified as a control using primers 5'-T7-AGA CGG TGG AGC CTG CGA TAA TG-3' and $5^{\prime}$-T7-AGA CGA CGG TGG AGG GGA GTC AA-3'. PCR products were purified with the MinElute PCR Purification Kit (Qiagen, Hilden, Germany), and $1 \mu \mathrm{g}$ of purified template DNA was used to produce dsRNA by in vitro transcription using the MEGAscript ${ }^{\circledR}$ RNAi kit (Ambion, Austin, USA) according to the manufacturer's instructions. RNA concentration was determined by spectrophotometry using the Nanodrop ND-1000 (peqlab, Erlangen, Germany), and RNA integrity was monitored by $1.4 \%$ denaturing agarose gel electrophoresis.

For RNAi experiments, approximately $0.1 \mu \mathrm{g}$ of the indicated dsRNA ( $1 \mu \mathrm{g} / \mu \mathrm{l})$ was injected laterally between abdominal segments 3 and 4 into pupae by using a nanoliter 2000 microinjector (WPI, Sarasota, USA). After injection, pupae were transferred to 6-cm Petri dishes containing basal medium with or without supplements.

Quantitative reverse transcription polymerase chain reaction (qRT-PCR)

Total RNA was extracted from 80 beetles per group using Trizol reagent (Invitrogen). One-step real-time RT-PCR was carried out in triplicate using $1 \mu \mathrm{l}$ of RNA template, the Brilliant II SYBR Green 1-step Q-RT-PCR-Kit and appropriate primers in a CFX 96 Real-Time PCR Detection System (BioRad, München, Germany). Changes in the target gene expression were calculated according to Pfaffl (2001) using equation $2^{-\Delta \Delta C T}$. For each sample, the fold 
change in the target gene was normalized to rpS18 RNA and was expressed in relation to control gene expression. To determine RNAi efficiency, unique primer pairs recognizing only cDNA derived from endogenous mRNA were designed to avoid cross-reaction between genomic DNA and the recombinant dsRNA. The primers for $\mathrm{rpS} 18$ were Tc-rpS18-F100 (5'-GCA AAG TCA TGT TTG CCC TCA C- $\left.3^{\prime}\right)$ and Tc-rpS18-B226 (5'-TTC TCG ACC TCT TCG TCG GAA C- $3^{\prime}$ ), those for $n r f-2$ were Tc-nrf-2-F1420 (5'-CAG CAC AAC CAT ACG TAC CAC C- $\left.3^{\prime}\right)$ and Tc-nrf-2-B1524 (5'-CAA ATG CTC CTC GTC ACC CTT C-3'), those for jnk-1 were Tc-jnk-1-F25 (5'-ACC GTT GAG GTG GGC GAC AC-3') and Tc-jnk-1-B201 (5'-CGC CCT TTT AGC GTG CGT CA-3'), those for foxo- 1 were Tc-foxo-1-F109 (5'-CCC AAC GAA GAG GGC AAC AAG TGC- $\left.3^{\prime}\right)$ and Tc-foxo-1-B217 (5'-GGT TGC CCC AGG CGT TCC GT- $3^{\prime}$ ), those for sirt- 1 were Tc-sirt-1F903 (5'-TCG GGA GCC TTT ACC GCA TTG- ${ }^{\prime}$ ) and Tc-sirt-1-B1008 (5'-GCC TTC CTT CCA AAC ACC GCC A-3') and those for sirt-3 were Tc-sirt-3-F190 (5'-TCC TGG GCT CGT CTT TAG TTG TCC-3') and Tc-sirt-3B5378 (5'-CCC CAA TCC ACG CAA CAT CCC G-3').

Relative mRNA levels compared to the control (imagos injected with IMPI dsRNA) were estimated in dsRNAinjected imagos after feeding with the control medium over 14 days at $32{ }^{\circ} \mathrm{C}$ and were $0.21 \pm 0.03(p<0.05)$ for $n r f$ $2, \quad 0.33 \pm 0.06 \quad(p<0.05) \quad$ for $j n k-1, \quad 0.10 \pm 0.02$ $(p<0.01)$ for foxo- $1,0.09 \pm 0.04(p<0.01)$ for sirt 1 and $0.16 \pm 0.04(p<0.01)$ for sirt-3.

\section{Microscopic analysis}

Beetles were analyzed using a MZ16F fluorescence stereomicroscope (Leica Microsystems, Wetzlar, Germany) equipped with DFC300FX Leica fluorescence camera. Beetles were fixed dorsally onto adhesive foil after folding the wings to allow the detection of muscle tissue fluorescence. Digital images were recorded using the Leica Application Suite software v2.8.1.

\section{Statistical analysis}

Statistical analysis was performed with GraphPad Prism software (v5.01, GraphPad, La Jolla, CA, USA) to present the longevity data as Kaplan-Meier survival curves. Survival curves were compared using the log-rank test with a significance threshold of $p<0.05$. Maximum life spans were determined as the average age of the oldest decile of surviving subjects. For statistical analysis of differences between two groups, a Student's unpaired $t$ test was used. For each variable, at least 3 independent experiments were carried out.

\section{Results}

Beetles were fed on a standard flour diet supplemented with $0.1,1,5$ or $10 \%$ lyophilized broccoli for 2 weeks and were tested against controls fed on a non-supplemented diet in a humidified chamber at $42{ }^{\circ} \mathrm{C}$. Under these conditions, the life span of beetles is considerably shorter than at $32{ }^{\circ} \mathrm{C}$ and the experiments are therefore completed more rapidly (Fig. 1). We found that the inclusion of broccoli increased the longevity of the beetles under heat-stress conditions, with the most pronounced effects observed when the diet contained $1 \%$ (Fig. 1b) and $5 \%$ (Fig. 1c) broccoli. The inclusion of $1 \%$ broccoli increased the average life span by $30.8 \pm 2.7 \%$ and the maximum life span by $32.7 \pm 3.5 \%(p<0.01)$ (Fig. 1b), whereas the inclusion of $5 \%$ broccoli increased the average life span by $24.7 \pm 3.1 \%$ and the maximum life span by $21.8 \pm 1.7 \%(p<0.01)$ (Fig. 1c). Food intake was neither affected at $32{ }^{\circ} \mathrm{C}$ (Fig. 2a) nor at $42{ }^{\circ} \mathrm{C}$ (Fig. 2b) by the supplementation of $0.1 \%$ or $10 \%$ broccoli to the diet when fed over 2,4 , or $6 \mathrm{~h}$, demonstrating that neither life span-extending nor reducing effects of broccoli were due to altered food intake.

Having established the positive impact of broccoli in the rapid thermotolerance assay, we also tested the effect of $1 \%$ broccoli on the longevity of beetles raised under normal physiological conditions $\left(32^{\circ} \mathrm{C}\right)$. We found that the life span curves matched those recorded under heat-stress conditions with regard to log-rank calculated differences and also with regard to maximal life span $(p<0.01)$, that is, broccoli also increased longevity in the absence of stress (Fig. 3).

The health-promoting characteristics of cruciferous vegetables have been attributed to the presence of isothiocyanates, so we tested the impact of major isothiocyanate compounds on the longevity of beetles by including the chemicals in the flour diet at the same concentrations found in the $5 \%$ lyophilized broccoli supplement. As shown in Fig. $4 a, 0.01 \%$ sulforaphane increased the life span, including the maximal life span of beetles significantly under heat-stress conditions $(p<0.01)$, although lower concentrations (5-, 10- and 100-fold reductions) had no impact (data not shown). Similarly, $0.01 \%$ phenylisothiocyanate increased the life span of beetles significantly under heat-stress conditions ( $p<0.01$, data not shown), although there was no effect at lower concentrations of $0.001 \%$, representing phenylisothiocyanate contents in the $5 \%$ broccoli diet (Fig. 4b). Allylisothiocyanate had no significant impact on longevity at $0.01 \%$ (data not shown) or at lower concentrations representing its contents in the $5 \%$ broccoli diet (Fig. 4c).

We used RNAi to identify the $T$. castaneum genes controlling longevity in response to broccoli and its 
Fig. 1 Broccoli enhances the longevity of $T$. castaneum under heat-stress conditions. KaplanMeier survival curves demonstrate the increased longevity of beetles fed on a broccoli-supplemented diet and then exposed to heat stress $\left(42{ }^{\circ} \mathrm{C}\right)$ compared to beetles fed on a control diet. Beetles were fed for 2 weeks on flour containing $0.1 \%$ (a), $1 \%$ (b), $5 \%(\mathbf{c})$ or $10 \%$ (d) of lyophilized broccoli. A log-rank (Mantel-Cox) test was used to determine the significance of differences between the survival curves. ${ }^{*} p<0.05$; $* * * p<0.001$. All experiments have been repeated more than once with similar effects
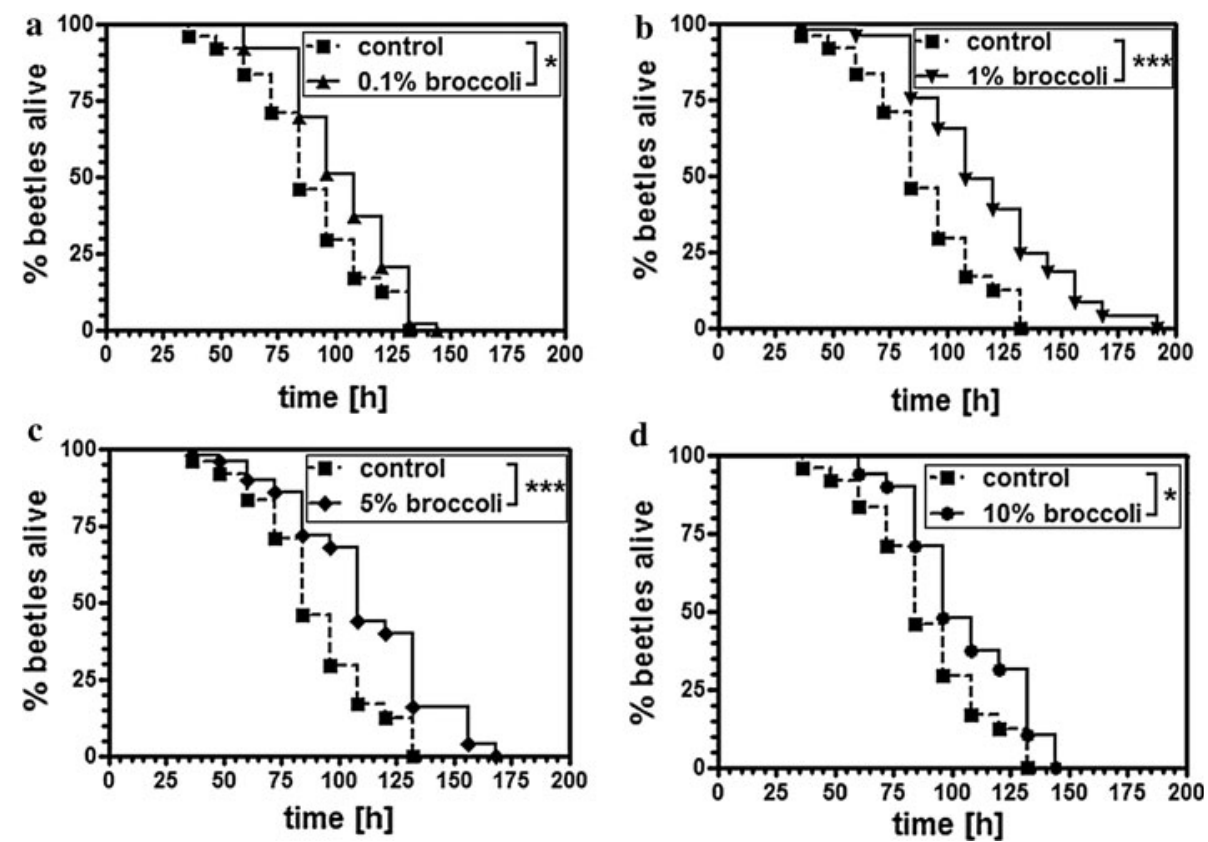
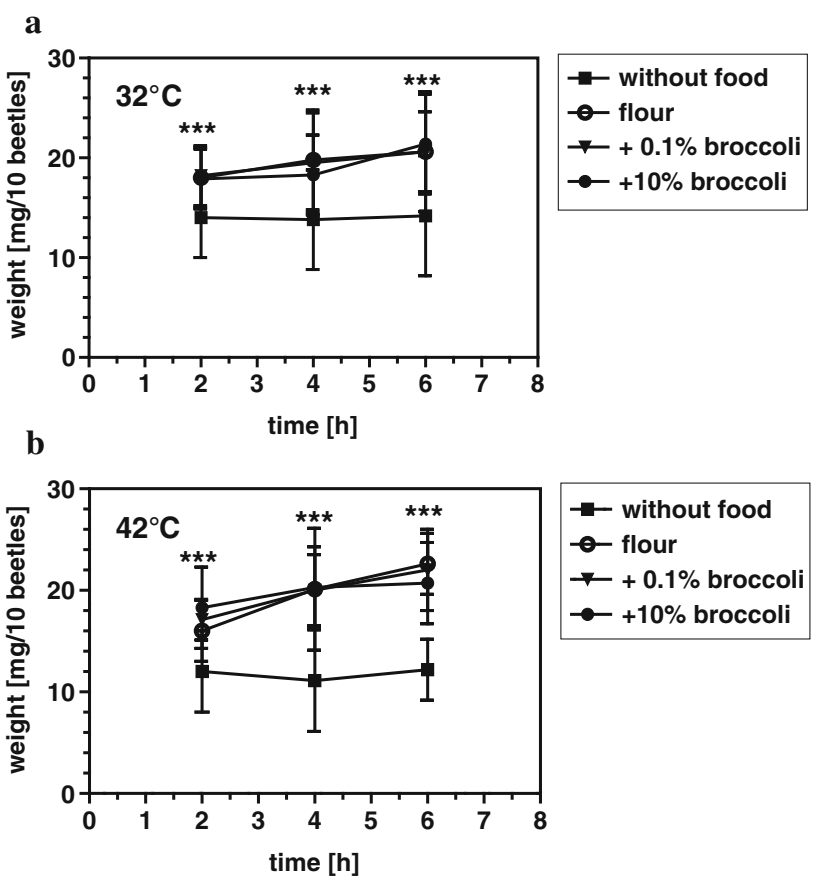

Fig. 2 Broccoli does not affect food intake. Beetles were starved for $6 \mathrm{~h}$ before they were fed on flour alone or supplemented with 0.1 or $10 \%$ broccoli or in the absence of food (control) either at $32{ }^{\circ} \mathrm{C}(\mathbf{a})$ or $42{ }^{\circ} \mathrm{C}(\mathbf{b})$. After 2,4 or $6 \mathrm{~h}$ individuals were frozen at $-18{ }^{\circ} \mathrm{C}$, and food intake was determined by measuring the wet weight of 10 pooled individuals per setting on a microbalance with 8 replicates per broccoli concentration and time point. $* * * p<0.001$ for beetles fed on flour \pm broccoli versus beetles in the absence of food

isothiocyanates. To test the efficiency of dsRNA administered in different ways, we used T. castaneum strain Pig19, which expresses green fluorescent protein (GFP) in

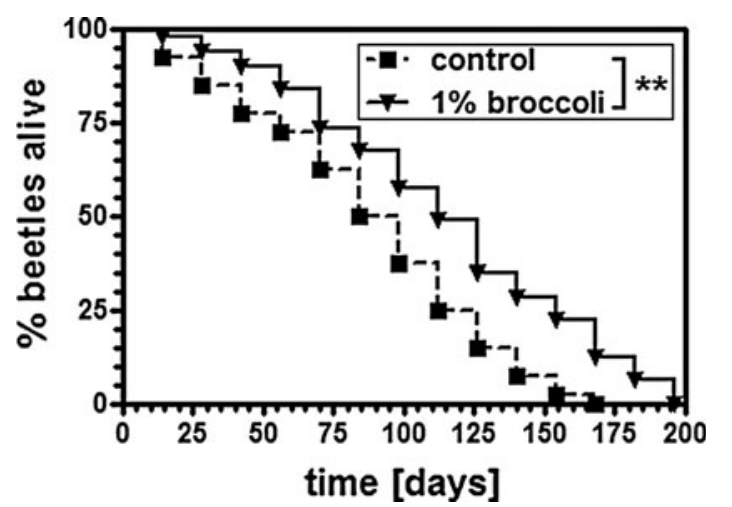

Fig. 3 Broccoli enhances the longevity of $T$. castaneum under normal physiological conditions. Kaplan-Meier survival curves demonstrate the increased longevity of beetles fed on a broccolisupplemented diet when reared at a normal physiological temperature $\left(32{ }^{\circ} \mathrm{C}\right)$ compared to beetles fed on a control diet. Beetles were reared on flour supplemented with $1 \%$ lyophilized broccoli from the young adult stage onwards. $* * p<0.01$

muscle tissue (Fig. 5). Feeding beetles with (GFP-)RNAiinducing bacteria, which is an effective strategy in $C$. elegans, did not reduce muscle fluorescence regardless of whether the bacteria were living (Fig. 5b) or lyophilized (Fig. 5c). Feeding beetles with in vitro transcribed dsRNA also had no effect on fluorescence (Fig. 5d). The microinjection of in vitro transcribed dsRNA into imagos caused a significant reduction in fluorescence (Fig. 5e), but by far the most effective strategy was the injection of dsRNA into pupae (Fig. 5f). This strategy was therefore chosen for the knockdown of candidate stress-response genes.

Isothiocyanates and particularly sulforaphane are potent phase II-inducing agents, so we investigated the hypothesis 
Fig. 4 The effect of selected isothiocyanates on the survival of $T$. castaneum at $42{ }^{\circ} \mathrm{C}$, based on their concentration in a normal diet supplemented with $5 \%$ lyophilized broccoli. The concentrations were set to $0.01 \%$ sulforaphane (a), $0.001 \%$

phenylethylisothiocyanate

(b) and $0.001 \%$

allylisothiocyanate (c).

$* * p<0.01$
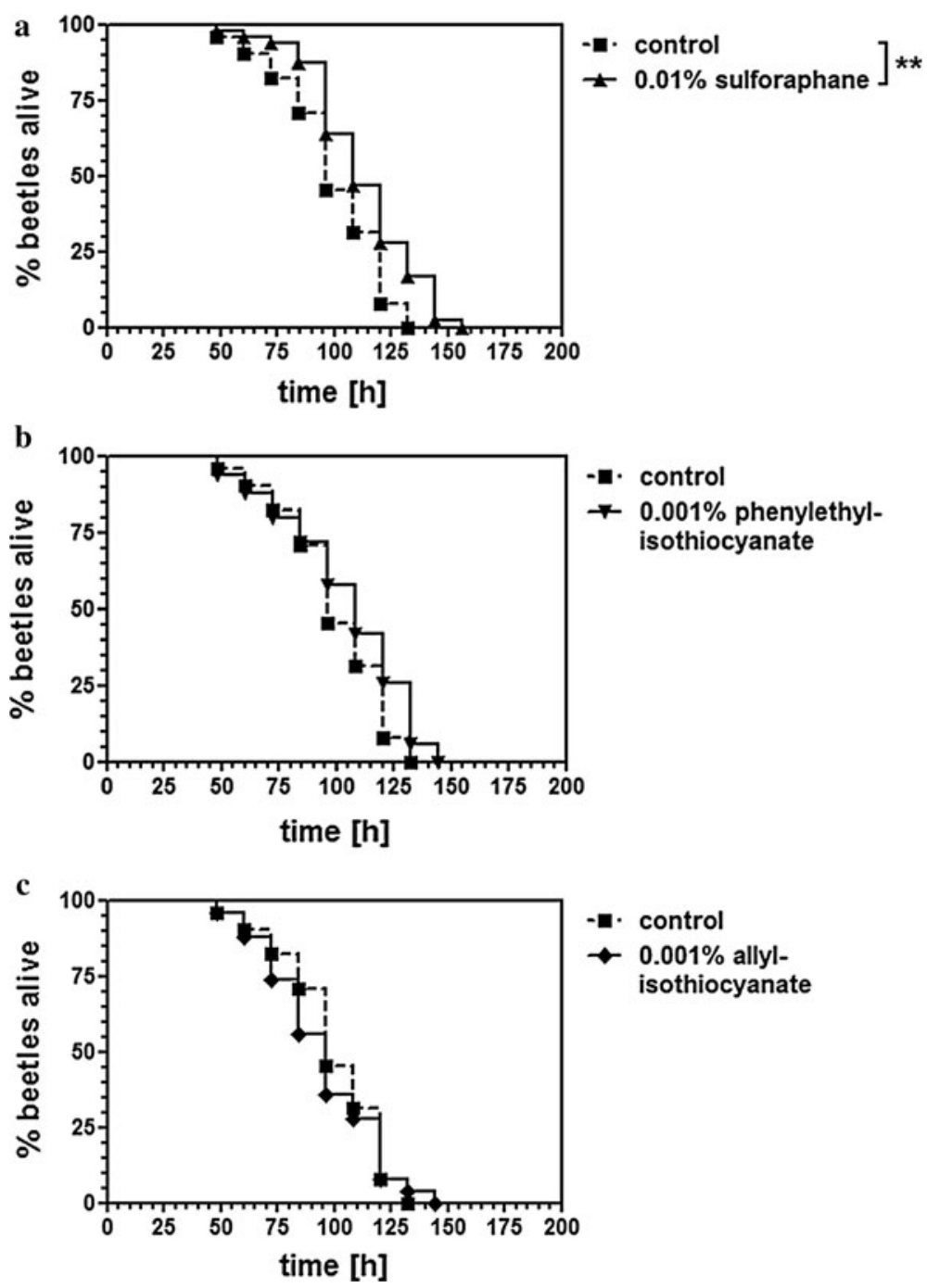

that broccoli and its high levels of isothiocyanates promote longevity by inducing xenobiotic metabolism and excretion. Accordingly, we targeted a beetle gene homologous to $n r f-2$, which encodes a key transcription factor required for the induction of phase II metabolism in mammalian cells. We found that reducing the abundance of $n r f-2$ transcripts by RNAi not only reduced the longevity of beetles under heat-stress conditions but also completely blocked the increase in longevity promoted by broccoli (Fig. 6a). We obtained similar results for the stress-activated kinase Jnk-1 (Fig. 6b) and the transcription factor Foxo-1 (Fig. 6c), which plays an important role in the regulation of adipogenesis, gluconeogenesis and glycogenolysis. In contrast, we found that the suppression of two sirtuin homologs (designated sirt-1 and sirt-3 based on their similarity to genes in other species) had no effect on longevity under heat-stress conditions and did not interfere with the beneficial effects of broccoli (Fig. 6d, e).

\section{Discussion}

Food habits play an important role in life expectancy, and the most effective and standardized intervention discovered thus far is caloric restriction. This involves reducing the energy intake from food by approximately $30 \%$ without limiting the supply of micronutrients and has been shown to slow the aging process in organisms as diverse as yeasts and primates (Colman et al. 2009). In humans, it is difficult to achieve compliance with caloric restriction in parts of the world where food is plentiful and access unlimited (Popkin et al. 2012). Researchers have therefore sought to identify compounds that activate the signaling pathways mediating the effects of caloric restriction to increase longevity without dietary restrictions (Howitz et al. 2003; Wood et al. 2004). Relevant pathways include those regulated by sirtuins $\left(\mathrm{NAD}^{+}\right.$-dependent deacetylases related to the yeast protein Sir2) which mediate several effects of caloric restriction (Cantó and Auwerx 2009; Qiu et al. 
Fig. 5 Microinjection of dsRNA into $T$. castaneum pupae induces efficient RNAi. GFP fluorescence in the T. castaneum reporter strain was visualized by fluorescence microscopy, with wings folded to reveal muscle fluorescence. Beetles were left untreated (a) or treated with dsRNA to knock down GFP either by feeding them with living RNAi bacteria (b), lyophilized RNAi bacteria (c), by feeding in vitro transcribed dsRNA (d) or by microinjecting in vitro transcribed dsRNA into the imagos (e) or the pupae (f). Images were captured $72 \mathrm{~h}$ after the treatments
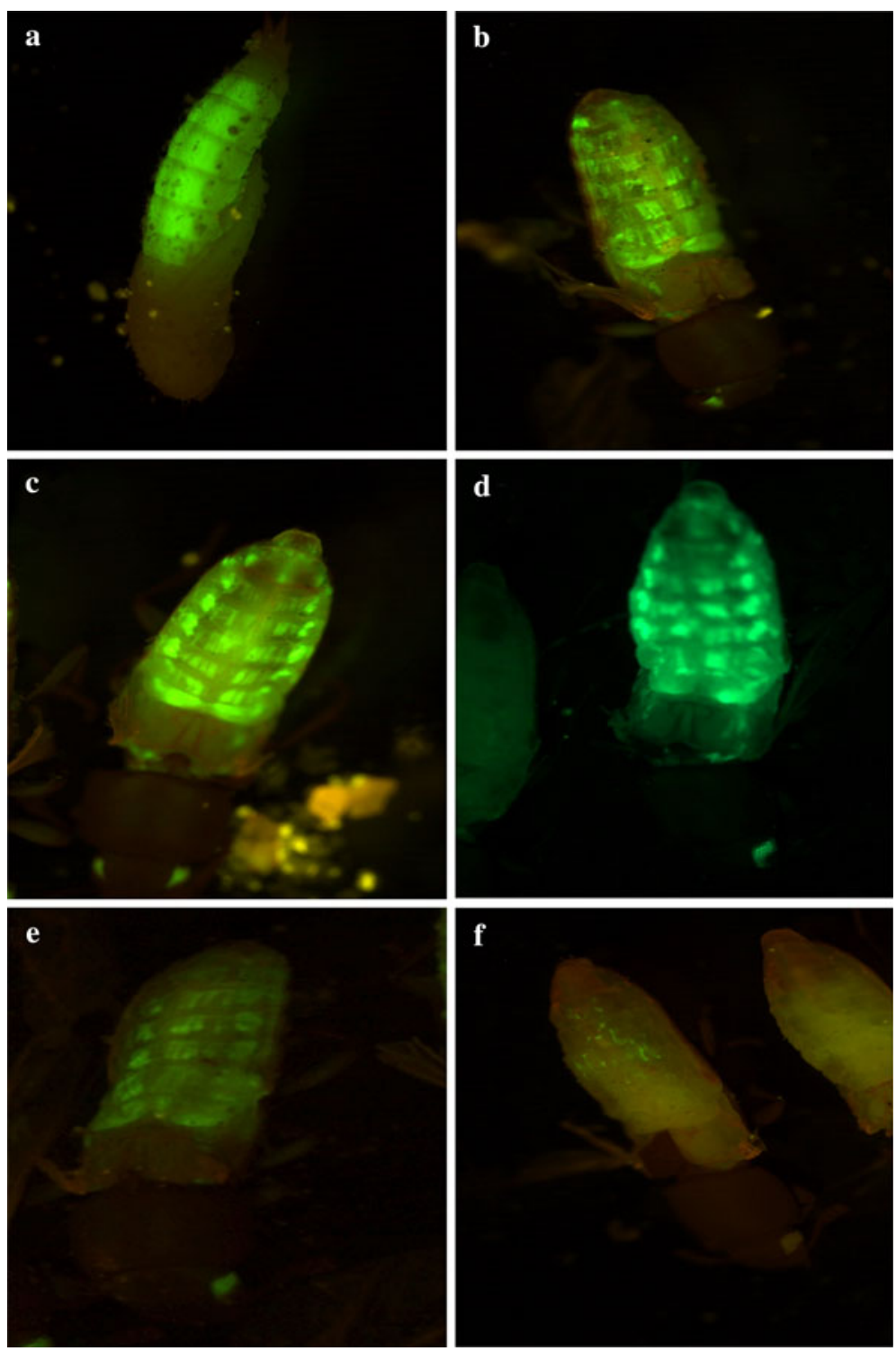

2010). Potent activators of sirtuins include resveratrol and fisetin, both found in red grapes and red wine, and quercetin, which is found in onions and apple skins (Howitz et al. 2003). Diets containing foods rich in these beneficial compounds are likely to achieve positive effects in humans, since, for example, resveratrol has already been shown to induce metabolic changes in obese individuals by mimicking the effects of caloric restriction (Timmers et al. 2011). However, it is difficult to assess the benefits of dietary interventions directly in humans under experimentally rigorous and ethical conditions, so inferences are drawn from indirect parameters that affect the human life span. For example, studies have shown that certain diets enriched in cholesterol accelerate the progression of cardiovascular disease, whereas diets rich in fruits and vegetables have the opposite effect. However, it is unclear whether these foods affect the age-related diseases specifically or the underlying aging process itself (Everitt et al. 2006).

These challenges can be addressed by developing simple experimental models that allow the impact of gene-food interactions on the aging process to be investigated within a convenient time frame. The most promising foods and active compounds can then be used as a basis to verify which are most efficient in more complex models. However, simple models such as $C$. elegans are limited because these organisms do not select and consume food in the same manner as humans, which means investigations are usually restricted to artificial diets spiked with single compounds rather than more complex foods. Based on our experiments, we propose the red flour beetle $T$. castaneum as a model organism for the investigation of gene-food interactions and their relevance in terms of stress resistance and aging. These beetles are advantageous first because 

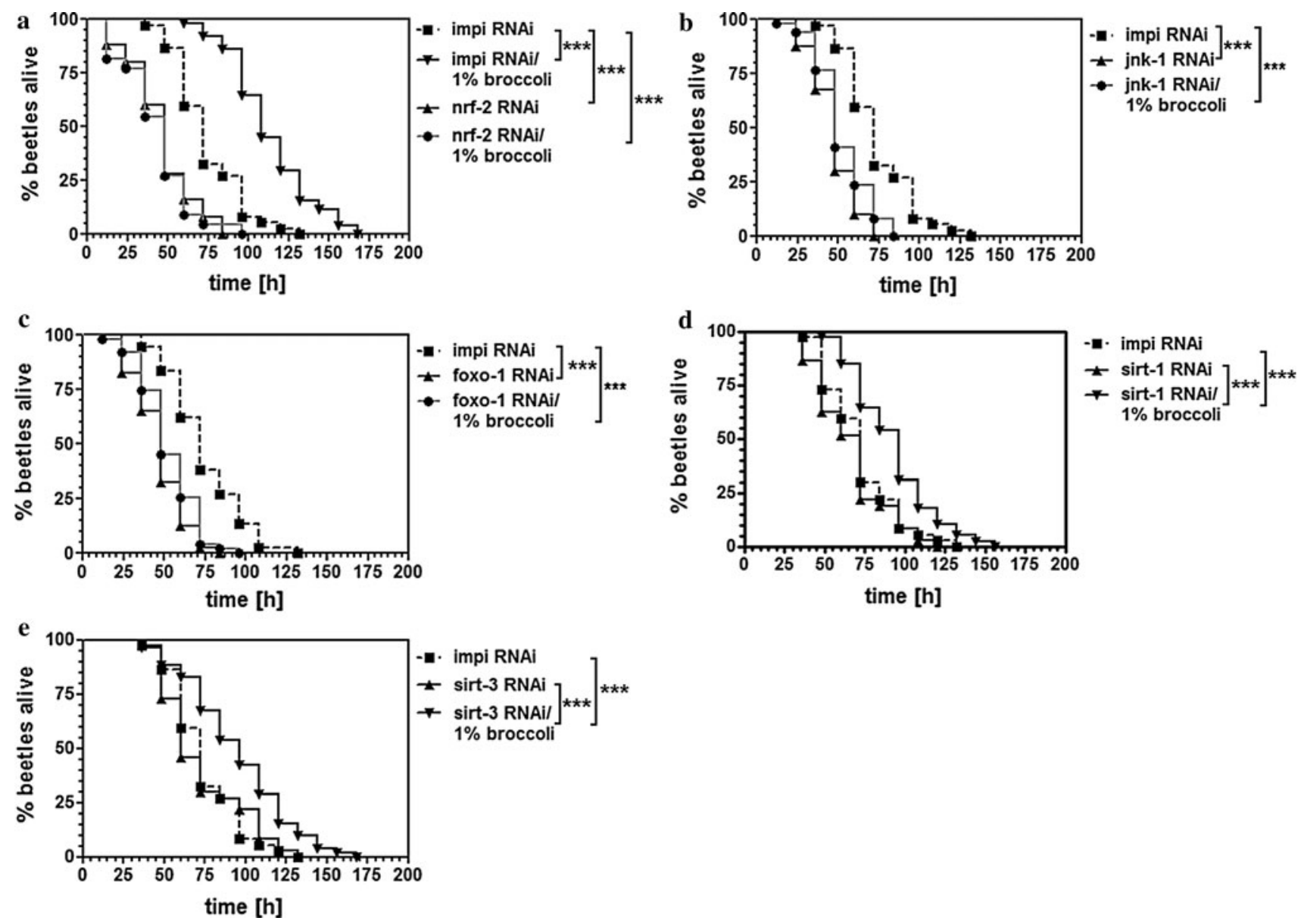

Fig. 6 Nrf-2 is necessary for the life-extending effects of broccoli. Transcripts encoding Nrf-2 (a), Jnk-1 (b), Foxo-1 (c), Sirt-1 (d) and Sirt-3 (e) were knocked down in T. castaneum by RNAi, achieved by

injecting dsRNA. Galleria mellonella IMPI was used as a control because there is no ortholog in T. castaneum. $* * * p<0.001$

they normally ingest dry flour, which means it is possible to mix flour with additional whole foods as long as the samples are lyophilized and second because the entire genome sequence is available (Richards et al. 2008), which makes it possible to use RNAi to test the roles of different genes and signaling pathways in stress resistance and aging.

We found that feeding the beetles on a flour diet supplemented with lyophilized broccoli increased their life span both under physiological conditions $\left(32^{\circ} \mathrm{C}\right)$ and under heat-stress conditions $\left(42{ }^{\circ} \mathrm{C}\right)$, with the greatest benefit achieved using 1-5\% broccoli. It cannot be predicted so far whether life span extensions observed at 32 and $42{ }^{\circ} \mathrm{C}$ are due to the same adaptations in response to broccoli feeding. Studies from other simple models, however, point to an important role of stress resistance in the determination of life span for cells and organisms (Calabrese et al. 2011; Rattan 2004). The health effects of broccoli have been investigated in humans, but as stated above it has only been possible to consider indirect

parameters, such as protection against cancer and diabetes. These studies have suggested that the protective effects result from the abundance of glucosinolate degradation products (such as the isothiocyanate compound sulforaphane) which appear to activate the Nrf-2-mediated induction of phase II detoxification enzymes that prevent oxidative damage and promote the removal of carcinogens (Juge et al. 2007). Indeed, the elevated expression of the transcription factor Nrf-2 can be regarded as an adaptive cellular stress response (Mattson 2008). When the stress stimulus is mild, the adaptive response may overcompensate for the stress and induce a state known as hormesis, in which the organism is protected from even normal or background levels of cellular damage that would accumulate in non-stressed individuals and contribute to the aging process (Kouda and Iki 2010).

Based on the above data and on previous reports showing that stress-response pathways are conserved between $T$. castaneum and mammals (Altincicek et al. 2008; Freitak et al. 2012; Knorr and Vilcinskas 2001), we 
proposed the hypothesis that similar mechanisms were responsible for the increased longevity of beetles fed on a broccoli-supplemented diet. Broccoli contains a maximum content of $10 \mu \mathrm{mol}$ glucoraphanin per gram of dry mass, and this is converted into sulforaphane by the enzyme myrosinase (Kushad et al. 1999). The molecular weight of sulforaphane is 177.3 ; therefore, the concentration of the myrosinase-released sulforaphane in a flour diet supplemented with $5 \%$ lyophilized broccoli (the maximum concentration promoting a highly significant increase in longevity) was estimated at $0.01 \%$. Accordingly, we found that the longevity of beetles fed on a flour diet artificially spiked with $0.01 \%$ sulforaphane was also increased significantly at $42{ }^{\circ} \mathrm{C}$. It is unlikely that sulforaphane alone is responsible for the antigeric effects of broccoli because diets spiked with a fivefold lower concentration $(0.002 \%)$ had no significant impact on beetle life span, whereas longevity increased significantly in beetles fed on diets supplemented with $1 \%$ broccoli (which contains the equivalent amount of sulforaphane). We tested two further isothiocyanates (phenylethylisothiocyanate and allylisothiocyanate) based on the abundance of the corresponding glucosinolates in broccoli florets (Kushad et al. 1999). We found that both were present at a maximum concentration of $0.001 \%$ in the $5 \%$ broccoli diet and that neither affected longevity at this concentration (although diets spiked with $0.1 \%$ phenylethylisothiocyanate did promote longevity). We therefore conclude that neither of these isothiocyanates is responsible for the increased longevity induced by broccoli.

We investigated the molecular mechanisms underlying the antigeric effects of broccoli by RNAi, which can be achieved in a wide range of insects by the injection of dsRNA (Price and Gatehouse 2008). RNAi can also be induced in insects by feeding them with dsRNA (a technique pioneered in C. elegans) but the efficiency of this strategy varies widely depending on the species (Price and Gatehouse 2008). We found that RNAi in T. castaneum was most efficient when dsRNA was injected into the pupae, as previously shown with Rhodnius prolixus (Araujo et al. 2006).

We initially targeted the T. castaneum Nrf-2 ortholog based on its key role in other species (Juge et al. 2007; Mattson 2008). We found that the knockdown of nrf-2 mRNA reduced the life span of insects in the thermotolerance test compared to untreated controls and completely abrogated the life span-increasing effects of broccoli. Nrf-2 therefore appears to be a key mediator of stress adaptation, longevity and hormesis in the red flour beetle. Likewise, knocking down the stress-activated kinase Jnk-1 or the forkhead transcription factor Foxo-1 also reduced the life span of beetles and abolished any beneficial effects of broccoli. Although there has been no direct evidence to show Nrf-2 regulates longevity, a cooperative interaction between Jnk-1 and Foxo-1 was previously shown to affect longevity in C. elegans (Oh et al. 2005). In so far, the genes identified to mediate the extension of survival due to broccoli feeding could be allocated to the so-called vitagenes, which are involved in preserving cellular homeostasis during stressful conditions (Calabrese et al. 2010, 2012). $\mathrm{Nrfs}$ and related pathways have been demonstrated in that context to integrate adaptive stress responses in the prevention of neurodegenerative diseases (Calabrese et al. 2010, 2012).

The ability of caloric restriction and certain plant secondary products to promote longevity and stress tolerance is mediated by sirtuins in C. elegans (Pietsch et al. 2011). We therefore identified sirtuin genes in the T. castaneum genome (designated sirt -1 and sirt-3 based on their closest relatives in other species) and carried out further RNAi experiments to suppress their activity. Interestingly, we found that in neither case was the longevity of beetles affected nor was there any impact on the life spanextending effects of broccoli. This indicates that individual T. castaneum sirtuins are not required to mediate the heatstress resistance and greater longevity induced by the consumption of broccoli.

In conclusion, the red flour beetle T. castaneum represents a valid model to address the interactions between genetic and environmental factors (such as diet) that contribute to stress tolerance and longevity. We have shown that Jnk-1 and Foxo-1, which have been associated with antigeric effects in C. elegans, are necessary for the increased longevity of beetles fed on diets supplemented with broccoli. Moreover, the transcription factor $\mathrm{Nrf}-2$, which increases stress resistance by inducing detoxification, was identified as a key regulator of longevity in beetles. The compounds and genes identified in our studies can now be investigated in more detail using more complex models.

Acknowledgments The authors acknowledge financial support from the Hessian Ministry of Science and Art (HMWK) via the collaborative research projects granted under the LOEWE programs "Insect Biotechnology" and "Translational Medicine and Pharmacology".

Conflict of interest None.

\section{References}

Altincicek B, Knorr E, Vilcinskas A (2008) Beetle immunity: identification of immune-inducible genes from the model insect Tribolium castaneum. Dev Comput Immunol 32:585-595

Anderson RM, Weindruch R (2012) The caloric restriction paradigm: implications for healthy human aging. Am J Hum Biol 24:101-106

Anderson RM, Shanmuganayagam D, Weindruch R (2009) Caloric restriction and aging: studies in mice and monkeys. Toxicol Pathol 37:47-51 
Araujo RN, Santos A, Pinto FS, Gontijo NF, Lehane MJ, Pereira MH (2006) RNA interference of the salivary gland nitrophorin 2 in the triatomine bug Rhodnius prolixus (Hemiptera: Reduviidae) by dsRNA ingestion or injection. Insect Biochem Mol Biol 36:683-693

Calabrese V, Cornelius C, Dinkova-Kostova AT, Calabrese EJ, Mattson MP (2010) Cellular stress responses, the hormesis paradigm, and vitagenes: novel targets for therapeutic intervention in neurodegenerative disorders. Antioxid Redox Signal 13:1763-1811

Calabrese V, Cornelius C, Cuzzocrea S, Iavicoli I, Rizzarelli E, Calabrese EJ (2011) Hormesis, cellular stress response and vitagenes as critical determinants in aging and longevity. Mol Aspects Med 32:279-304

Calabrese V, Cornelius C, Dinkova-Kostova AT, Iavicoli I, Di Paola R, Koverech A, Cuzzocrea S, Rizzarelli E, Calabrese EJ (2012) Cellular stress responses, hormetic phytochemicals and vitagenes in aging and longevity. Biochim Biophys Acta 1822:753-783

Cantó C, Auwerx J (2009) Caloric restriction, SIRT1 and longevity. Trends Endocrinol Metab 20:325-331

Chen D, Guarente L (2007) SIR2: a potential target for calorie restriction mimetics. Trends Mol Med 13:64-71

Colman RJ, Anderson RM, Johnson SC, Kastman EK, Kosmatka KJ, Beasley TM, Allison DB, Cruzen C, Simmons HA, Kemnitz JW (2009) Caloric restriction delays disease onset and mortality in rhesus monkeys. Science 325:201-204

Dinkova-Kostova AT, Holtzclaw WD, Cole RN, Itoh K, Wakabayashi N, Katoh Y, Yamamoto M, Talalay P (2002) Direct evidence that sulfhydryl groups of Keap1 are the sensors regulating induction of phase 2 enzymes that protect against carcinogens and oxidants. Proc Natl Acad Sci USA 99:11908-11913

Everitt AV, Hilmer SN, Brand-Miller JC, Jamieson HA, Truswell AS, Sharma AP, Mason RS, Morris BJ, Le Couteur DG (2006) Dietary approaches that delay age-related diseases. Clin Interv Aging 1:11-31

Fontana L, Klein S (2007) Aging, adiposity, and calorie restriction. JAMA 297:986-994

Fontana L, Partridge L, Longo VD (2010) Extending healthy life span-from yeast to humans. Science 328:321-326

Freitak D, Knorr E, Vogel H, Vilcinskas A (2012) Gender and stressor specific miRNA expression in Tribolium castaneum. Biol Lett 8:860-863

Frescas D, Valenti L, Accili D (2005) Nuclear trapping of the forkhead transcription factor FoxO1 via Sirt-dependent deacetylation promotes expression of glucogenetic genes. J Biol Chem 280:20589-20595

Howitz KT, Bitterman KJ, Cohen HY, Lamming DW, Lavu S, Wood JG, Zipkin RE, Chung P, Kisielewski A, Zhang LL, Scherer B, Sinclair DA (2003) Small molecule activators of sirtuins extend Saccharomyces cerevisiae lifespan. Nature 425:191-196

Juge N, Mithen RF, Traka M (2007) Molecular basis for chemoprevention by sulforaphane: a comprehensive review. Cell Mol Life Sci 64:1105-1127

Knorr E, Vilcinskas A (2001) Post-embryonic functions of HSP90 in Tribolium castaneum include regulation of compound eye development. Dev Genes Evol 211:357-362

Kouda K, Iki M (2010) Beneficial effects of mild stress (hormetic effects): dietary restriction and health. J Physiol Anthropol 29:127-132

Kushad MM, Brown AF, Kurilich AC, Juvik JA, Klein BP, Wallig MA, Jeffery EH (1999) Variation of glucosinolates in vegetable crops of Brassica oleracea. J Agric Food Chem 47:1541-1548
Lee YJ, Ducoff HS (1983) Age and sensitivity to oxygen in the flour beetle, Tribolium confusum. Mech Ageing Dev 22:97-103

Lewis SM, Tigreros N, Fedina T, Ming QL (2012) Genetic and nutritional effects on male traits and reproductive performance in Tribolium flour beetles. J Evol Biol 25:438-451

Lorenzen MD, Berghammer AJ, Brown SJ, Denell RE, Klingler M, Beeman RW (2003) piggyBac-mediated germline transformation in the beetle Tribolium castaneum. Insect Mol Biol 12:433-440

Masoro EJ (2005) Overview of caloric restriction and ageing. Mech Ageing Dev 126:913-922

Mattson MP (2008) Dietary factors, hormesis and health. Ageing Res Rev 7:43-48

McWalter GK, Higgins LG, McLellan LI, Henderson CJ, Song L, Thornalley PJ, Itoh K, Yamamoto M, Hayes JD (2004) Transcription factor Nrf2 is essential for induction of $\mathrm{NAD}(\mathrm{P}) \mathrm{H}$ :quinone oxidoreductase 1, glutathione S-transferases, and glutamate cysteine ligase by broccoli seeds and isothiocyanates. J Nutr 134:3499S-3506S

Oh SW, Mukhopadhyay A, Svrzikapa N, Jiang F, Davis RJ, Tissenbaum HA (2005) JNK regulates lifespan in Caenorhabditis elegans by modulating nuclear translocation of forkhead transcription factor/DAF-16. Proc Natl Acad Sci USA 102:4494-4499

Pfaffl MW (2001) A new mathematical model for relative quantification in real-time RT-PCR. Nucleic Acids Res 92:2002-2007

Pietsch K, Saul N, Chakrabarti S, Stürzenbaum SR, Menzel R, Steinberg CE (2011) Hormetins, antioxidants and prooxidants: defining quercetin-, caffeic acid- and rosmarinic acid-mediated life extension in C. elegans. Biogerontology 12:329-347

Popkin BM, Adair LS, Ng SW (2012) Global nutrition transition and the pandemic of obesity in developing countries. Nutr Rev 70:3-21

Price DR, Gatehouse JA (2008) RNAi-mediated crop protection against insects. Trends Biotechnol 26:393-400

Qiu X, Brown KV, Moran Y, Chen D (2010) Sirtuin regulation in calorie restriction. Biochim Biophys Acta 1804:1576-1583

Rattan SI (2004) Hormetic mechanisms of anti-aging and rejuvenating effects of repeated mild heat stress on human fibroblasts in vitro. Rejuvenation Res 7:40-48

Richards S, Gibbs RA, Weinstock GM, Brown SJ, Denell R, Beeman RW, Gibbs R, Beeman RW, Brown SJ, Bucher G et al (2008) Tribolium genome sequencing consortium. The genome of the model beetle and pest Tribolium castaneum. Nature 452:949955

Ristow M, Zarse K, Oberbach A, Klöting N, Birringer M, Kiehntopf M, Stumvoll M, Kahn CR, Blüher M (2009) Antioxidants prevent health-promoting effects of physical exercise in humans. Proc Natl Acad Sci USA 106:8665-8670

Soliman MH (1987) Ageing and parental age effects in Tribolium (review). Arch Gerontol Geriatr 6:43-60

Son TG, Camandola S, Mattson MP (2008) Hormetic dietary phytochemicals. NeuroMol Med 10:236-246

Timmers S, Konings E, Bilet L, Houtkooper RH, van de Weijer T, Goossens GH, Hoeks J, van der Krieken S, Ryu D, Kersten S (2011) Calorie restriction-like effects of 30 days of resveratrol supplementation on energy metabolism and metabolic profile in obese humans. Cell Metab 14:612-622

Wood JG, Rogina B, Lavu S, Howitz K, Helfand SL, Tatar M, Sinclair D (2004) Sirtuin activators mimic caloric restriction and delay ageing in metazoans. Nature 430:686-689 